\title{
Prevalence of Known Risk Factors in Women Diagnosed with Breast Cancer at Inmol Hospital, Lahore, Punjab
}

\author{
Muhammad Mansha1*, Maryam Saleem¹, Muhammad Wasim², Muhammad \\ Tariq $^{1}$
}

\begin{abstract}
Background: Breast cancer is the most prevalent cancer in women worldwide and its frequency is increasing gradually in many countries. Over the last three decades an increase in the breast cancer has been witnessed in the earlier low-risk Asian countries including Pakistan. Purpose: The objective of the current study was to assess the prevalence of known risk factors like early menarche, late menopause, socio economic, reproductive and demographic factors, among women diagnosed with breast cancer at INMOL hospital, Lahore, Punjab, as little information exists in this regard. Materials and Methods: A survey study was conducted on 200 women diagnosed with breast cancer who were seen at Institute of Nuclear Medicine and Oncology (INMOL) hospital, Lahore. A structured questionnaire was administered to these patients regarding the known risk factors through face to face interviews after obtaining appropriate consent. Results: Regarding non-modifiable risk factors, our study showed that majority of the breast cancer patients were diagnosed at $35-45$ years $(32.5 \%)$ or at older age ( $\leq 46)$ and experienced menarche at 12 years or older $(66 \%)$. Likewise, a large number of patients reached menopause at the age of 45 years $(60 \%)$, had no family and personal history of breast cancer $(80 \%)$ and hence fell in a low risk category. Regarding modifiable risk factors in women diagnosed with breast cancer, most of the patients fell in low risk strata as the majority were married $(\mathbf{9 8 \%})$ at young age, breastfed their children for 12 months or more $(\mathbf{8 8 \%})$ ) and bore two to three children $(80 \%)$. Considering income criteria, the majority of the patients had a low risk profile as they belonged to middle class $(70 \%)$, urban area $(60 \%)$ and were house wives $(\mathbf{8 0 \%})$. However, it was noted that a considerable number of women $(34 \%)$ diagnosed with breast cancer experienced menarche at an early age $(<12)$ and reached menopause after the age of 45 years. This situation is further augmented by environmental changes and dietary habits and places them in a high risk category.
\end{abstract}

Keywords: Breast cancer - risk factors - menarche - menopause - demographic factors - INMOL hospital Lahore

Asian Pac J Cancer Prev, 17 (2), 563-568

\section{Introduction}

Breast cancer is the most wide spread cancer globally and predominantly affects the lobules (milk producing glands) and milk ducts of breast which carry milk to the nipples (Sariego, 2010). Many women die every year due to breast cancer in Asia and Latin America and now it is being considered the prominent cancer worldwide among women (Saweer et al., 2003) both in the industrialized and the developing countries (Sasco, 2001; Saweer et al., 2003; Porter, 2009). Globally, breast cancer, due to its high prevalence, has emerged as 2 nd most common cancer in the world and signifies $9 \%$ of universal cancer burden (Parkin et al., 2002). It is affecting 1.38 Million individual globally every year (Lee et al., 2012). In developing countries breast cancer is targeting the women at an early age with severe biological characteristics (Bhikoo et al., 2011).

In western industrialized societies breast cancer usually upsets women living who are between the ages of 35 and 70 but it can also hit young women. The quantum and frequency of breast cancer is quite high in Western Europe and lowest in Eastern and Middle Africa, with approximately five-time difference globally (Ferlay et al., 2008). The highest incidence rate among the countries of European Union in 2008 was estimated around 145 cases per 100,000 in Belgium and 57 cases per 100,000 in Greece (Sasieni et al., 2011).

During 1993-1997, in China, the frequency of breast cancer was rated at 27.2 per 100000 in urban Shanghai whereas in rural area like Qidong, it was just 11.2 per 100000 (Parkin et al., 2005; Chen et al., 2006). Similarly, two-fold variation in breast cancer incidence was observed between Seoul (20.8 per 100000) and more rural Kanghwa county (12.7 per 100000) in Korea (Parkin et al., 2005).

Among Asian countries especially in Pakistani population there has been a shocking escalation in the prevalence of breast cancer. Although in Pakistan there is 
no organized data pertaining to breast cancer incidence, mortality and risk factor available but there were reports that breast cancer accounts for $34.6 \%$ of all female cancers. The prevalence of breast cancer in Pakistan is 2.5 times greater than bordering countries; Iran and India, (Mousavi et al., 2006), reflecting the highest frequency of breast cancer for any Asian population, after Jews in Israel (Bhurgri et al., 2000; Liede et al., 2002). In Pakistan, the frequency of breast cancer is "50 per 100,000 ", which is quite high when compared to Indian women, "19 per 100,000” (Kumar et al., 2009). According to Karachi Cancer Registry, the age-standardized incidence rate (ASR) for breast cancer (KS) for period 1995- 1997 was $51.7 / 100,000$ in Karachi South which was one third of all female cancers (Bhurgri et al., 2004).

The present study was carried out to assess the prevalence of known risk factors among women diagnosed with breast cancer who regularly visited INMOL hospital, Lahore for follow up and treatment. A structured questionnaire was made to collect the information pertaining to known risk factors; (non-modifiable) like personal and family history of disease, age at diagnosis, age at first menarche and menopause, (and modifiable) socio-demographic characteristics and reproductive factors. The obtained data was analyzed and the results reflected that majority of the patient fell in low risk strata but the environmental factors and dietary habits augment the disease day by day and it is dire need to address this menace on government and public level by creating awareness and taking immediate measures. The results also emphasis the women to take care of their health because early detection of the disease will enhance the survival of the patients greatly.

\section{Materials and Methods}

\section{Study design}

The current work was a hospital based descriptive cross sectional study and was undertaken at Institute of Nuclear Medicine of Oncology Lahore (INMOL) Punjab,Pakistan. The study was conducted for one year from june 2013 to May 2014. The study population comprised of 199 females and 1 male breast cancer patients presented during the study period who had positive histological results.

The risk factors that were evaluated in the present study were categorized in to non-modifiable and modifiable risk factors for breast cancer. The non-modifiable factors consists of age at diagnosis, history of breast cancer and menstrual history.The history of breast cancer indicates whether a patient had a family member with the disease, either mother, sister or aunt (Wardlaw, 2012). Similarly menstrual history reflects the age of women at menarche and menopause.

Modifiable risk factors comprise, socio-demographic aspects like marital status, place of living, life style habits (smoking, alcohol intake, level of education and income) and reproductive factors (child bearing history, age at first pregnancy and breast feeding).

\section{Quality control}

Data was collected through planned face to face interview in a separate sitting place. The researcher clarified the purpose of the questionnaire to the women before attaining consent. Great care was taken to ensure the secrecy; and enough time was given to the patients for answering the questions and encouraged them to be open in answering. During the interview any intricate information was simplified to get exact and clear answer by the patients.With the exception of few non modifiable factors, all other modifiable factors had multiple choice response like yes or no or $1 / 2 / 3 / 4$.

\section{Questionnaire contents}

The data was collected through in-person interview based on structured questionaire from the breast cancer patients. The data mainly covered risk factors of breast cancer: (i) socio-demographic factor: age at diagnosis, marital status, level of education, socio-economic status. (ii) Female physiological and reproductive factors: age at menarche, age at menopause, child bearing history, lactation history (iii) Breast cancer history (iv) life style habits: smoking (ever / never smoking), alcohol intake (ever/ never used alcohol).

Socio-economic status was determined by relating the information on income and occupation of self / husband or parents. Those who had some regular official source of income with an income of rupee twelve thousands or below were coded as 'lower', those who had income between twelve to thirty five thousands were considered 'middle' and otherwise coded as 'upper'.

The interviewer directed a questionnaire to patients who were waiting to be examined by physician or admitted in wards. Information collected is then entered into questionnaires. An identification key, including each patient's name, residence, age, date of visit to the hospital was used for linkage. Cases were interviewed once.

\section{Results}

\section{Age at diagnosis with breast cancer}

The age at diagnosis of breast cancer was divided into five age groups. Out of 200 women diagnosed with breast cancer, $32.5 \%$ patients belonged to $35-45$ years of age and hence were stratified in high risk profile. Wheare as $33 \%$ cases were diagnosed at 46-56 year of age and belong to low risk profile. Our results also showed that $17.5 \%$ cases were at high risk of breast cancer development becuase they were diagnosed at less than 35 years of age. Moreover $14 \%$ cases were diagnosed between 57-65 years (Figure 1).

\section{Age at menarche and menopause}

Out of 200 patients, majority of the women $(65.5 \%)$ experienced their first menarche at 12 years or older and were at low risk of breast cancer development wheareas $34.5 \%$ patients underwnet menstrual periods at less than 12 years and hence were stratified into high risk cancer development. $61 \%$ cases reached menopause at or below the age of 50 years and belonged to low risk profile but a considerable number of cases $(39 \%)$ had their menopause after 50 years and palced the woman into high risk profile (Figure 2). 
Incidence of Breast cancer and Family History

Family history of breast cancer is a critical risk factor. About $20 \%$ patients had family history of breast cancer which corressponds with high risk for breast cancer development where as $80 \%$ of the patients were suffered from the breast cancer with out any personal and family

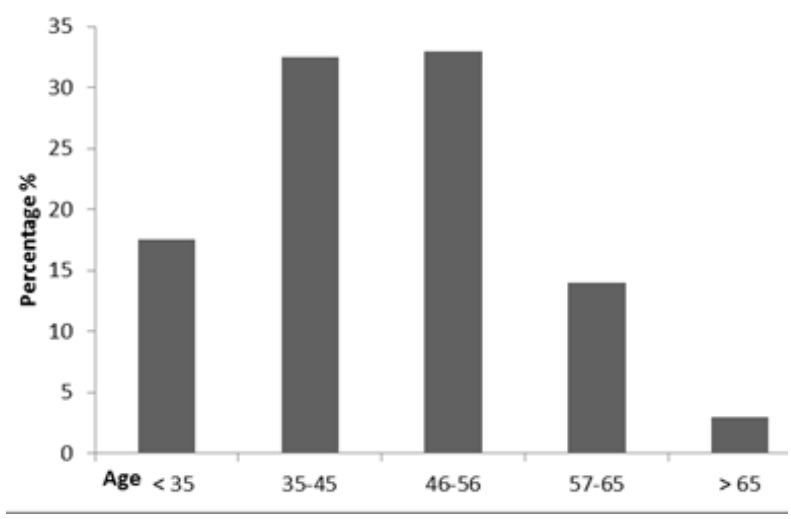

Figure 1. 200 Breast Cancer Patients, who Visited/ Admitted to INMOL Hospital, were Analyzed with Respect to age at Diagnosis. Breast cancer patients included in this study were categorized in to five groups basing on the age at diagnosis (age below 35 and greater than 65 years)

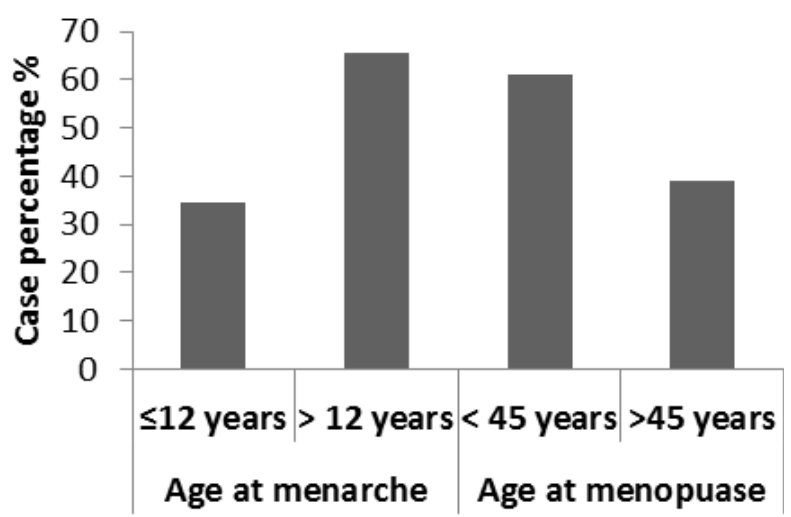

Figure 2. 200 Breast Cancer Patients, who Visited/ Admitted INMOL Hospital, were Analyzed with Respect to age at Menarche and Menopause. Greater number of patients can be observed at early menarche and late menopause

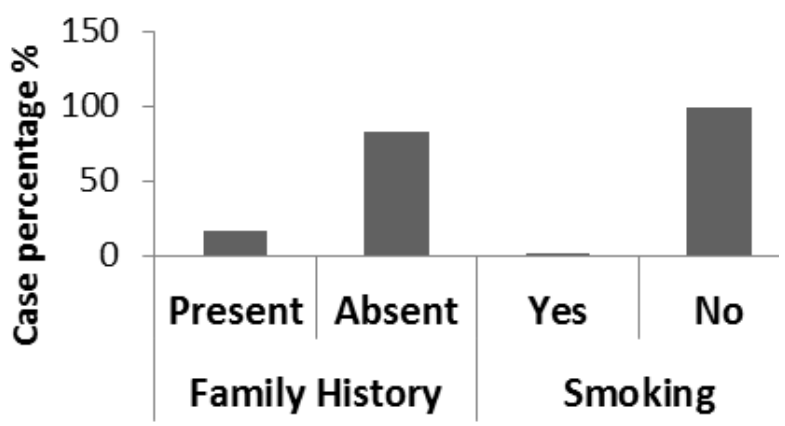

Figure 3. 200 Breast Cancer Patients, who Visited/ Admitted INMOL Hospital, were Evaluated with Respect to Family History of Cancer and Smoking. The results are specified in terms of number of cases in percentages history of any type of cancer. Alcohol intake and Smoking history does not appear to be a risk factor in current population as only $1.5 \%$ of patients were found smokers. The majority of patients $(98.5 \%)$ were non-smokers and fell in a low risk profile (Figure 3).

\section{Breast cancer and socio-demographic variables}

Socio-demographic profiles are considered important in the prevelance of breast cancer. To ascertain any link between breast cancer and socio-demographic status, different risk factors like marital status, residence and income were taken in to consideration.

\section{Marital status and breast cancer}

Regarding marital status of the breast cancer pateints, most of the cases $(96.5 \%)$ belong to married women and hence corresspond with low risk breast cancer developmenat. Only $3.5 \%$ cases fell in high risk strata being unmarried. It is also observed that unmarried women belonged to age group less than 35 years and had high risk of breast cancer. Further, the living area poses risk in breast cancer development. The data indicated that $59.5 \%$ breast cancer women belonged to urban area and were more vulnerable to cancer than women from rural areas $(41.5 \%)$, which fell in medium risk factor category. Moreover, the data reflected that a substantial number of

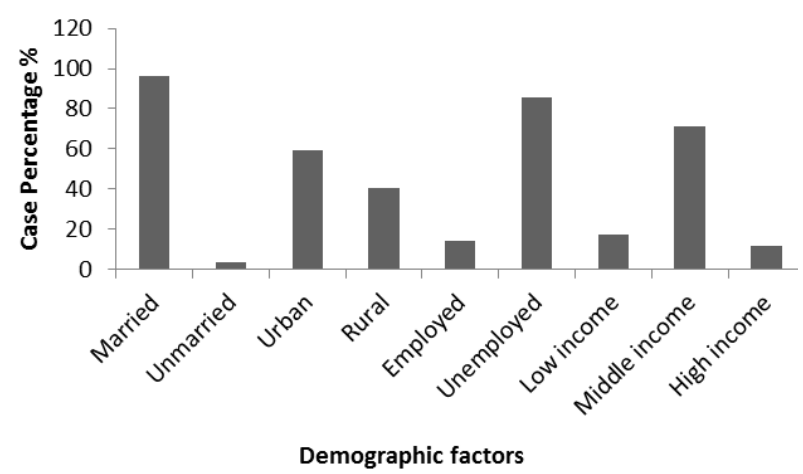

Figure 4. 200 Breast Cancer Patients, who Visited/ Admitted to INMOL Hospital, were Analyzed with Respect to Demographic Factors. Breast cancer patients included in this study were categorized with respect to marital status, residential areas and financial status

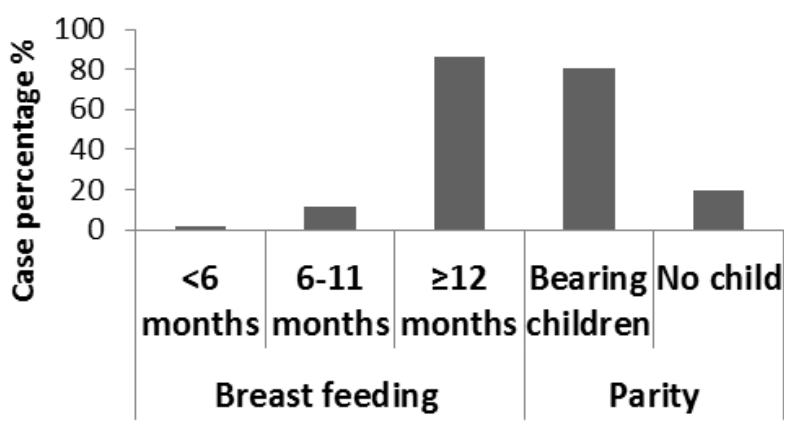

Figure 5. 200 breast cancer patients, who visited INMOL hospital, were analyzed with respect to breast feeding and Parity. The results are designated in terms of percentage 


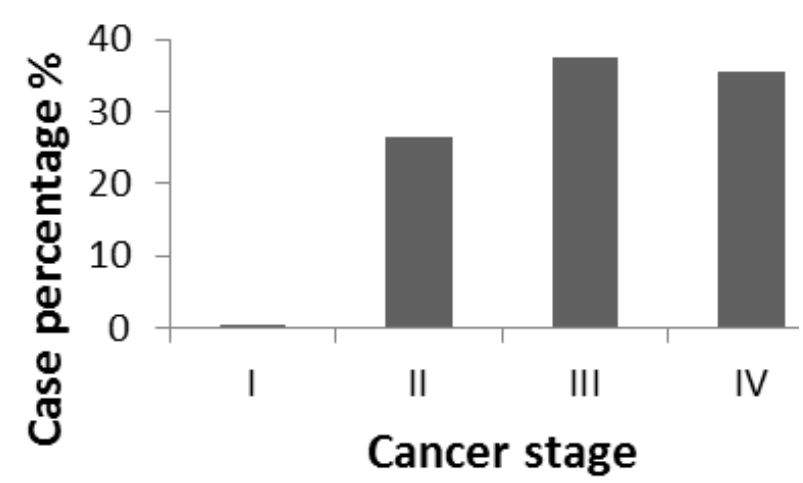

Figure 6. 200 Breast Cancer Patients, who Visited/ Admitted to INMOL Hospital, were Evaluated with Respect to Cancer Stage. The results are specified in terms of number of cases in percentages

the patients $(85.5 \%)$ did not belong to any working class (house wives) and hence fall in low risk profile (Figure 4).

Incidence of breast cancer with relation to breast feeding and parity

Breast feeding and parity are critical risk factors of breast cancer. The results revealed that only $2 \%$ patients were those who didn't breast feed or breast feed their children for a very short time period. Most of the women $(88 \%)$ in the existing study breast feed their children for 12 months or more and hence fall in a low risk strata. Similarly when the data was examined with relation to parity, it was found that $20 \%$ patient did not bear children and hence were at critical risk factor in breast cancer development (Figure 5).

Incidence of Breast cancer with relation to cancer stage

All the patients (200) were categorized with respect to biological severity and cancer stages. The current study revealed that a negligible number of cases were diagnosed at stage 1 where as most cases of breast cancer belonged to stage III and IV (i.e., $37.5 \%$ and 35.5\%). A substantial number $(26.5 \%)$ of cases were identified at stage II (figure 6).

\section{Discussion}

Age at diagnosis: In this study 200 breast cancer patients were interviewed with respect to prevelence of different risk factors who visited/ treated at INMOL hospital Lahore. The study reflected certain risk factors as critical one which augmented the development and escalation of beast cancer.

Our analysis showed that the youngest patient in this study was 21 years old and oldest one was 80 years old. $17.5 \%$ of the patients were diagnosed before the age of 35 years whereas majority $(82.5 \%)$ of the patients in the present study were 46 years of age or even older at diagnosis. This data is compatible to the findings of Rambau (2011) who reported that the median age at diagnosis among Tanzanian women was 46 years, and $32.5 \%$ cases were diagnosed at $35-45$ years age. Regarding the stratification of age at diagnosis our studies were in agreement to the the Tanzanian (Rambau et al. 2011) and Nigerian (Ogundiran et al., 2010) studies. Moreover, the age at the time of diagnosis reflects the quantum of danger because once a woman develops a primary tumor, the chances of developing a secondry tumor enhances in her breast (Chen et al., 1999). It means that screening for breast cancer should be performed at an early age for earlier detection and therapy.

Age at menarche and menopause: Our results reflected that majority $(65.5 \%)$ of the patients exprerienced their first menarche at an age of 12 years and older and hence were placed at low risk level. Women who suffered menarche at younger age ( $\leq 12$ years) have high risk of evolving breast cancer since prolonged menstrual history multiplies woman contact to oestrogen (Sprague et al., 2008).

The average age at menarche for the investigated patients was 13 years, this result equals to the Californian patients, whose average age at menarche was 12.6 years (Wrensch et al., 2003). In the present study $34.5 \%$ had early menarche ( $\leq 12$ years) which contrasted to the data of Northwest Ethopian women whose age at menarche was 15.8 \pm 1 year (Zegeye et al. 2009). There are reports that indicate that African women had menarche at older ages and hence were at low risk of breast cancer (Fregene and Newman, 2005). This suggests that interaction between genetic and environmental factors determin the age at menarche (Karapanou and Papadimitriou, 2010).

Contrary to the menarche, women who reach menopause after 50 years of age are more prone to evolve breast cancer because of long exposure to estrogens (Clemons et al., 2001). Our results indicated that 39\% women experienced menopause after the age of 45 years and this is in agreement with the data of AfricanAmericans women who underwent menopause at older age (Berstad et al., 2010) and were placed at high risk category.

Family history: It is reported that women with family history of cancer has more tendency to develop cancer but in the present study majority of the patients $(83 \%)$ did not have any relatives diagnosed with cancer and were stratified in low risk level. Only $17 \%$ of the patients in our sample study had first degree relatives with cancer which stratified them at high risk level. But these findings are contrary to the study conducted in India, where $85.0 \%$ patients had relatives with breast cancer (Puri et al., 2009). Moreover, it is reported that Breast Self-Examination reduces the cancer worries and imporves survival in women with family history of cancer (Norman et al., 2005). Most of the African women did not share any information regarding family history of cancer because of decreased awareness and their desire to keep this information secret (Anyanwu, 2000). Some of the patients in the existing study also specified that only their close relatives (father and mother) knew about their disease. It imlpies that in asia and africa the patients tend to keep their disease secret and become victim of serious cirsumstances. Moreover, it is reported that there is a suspicious link between between smoking and breast cancer and it is observed that presentation of breast cancer occurs eralier in passive and chain smokers as compared to non smokers (Abhidha Malik., 2015). Our results are 
in agreement with this notion that our $98.5 \%$ patients fall in low risk category.

Marital status itself does not indicate being risk of breast cancer (Abbasis et al., 2009) as majority of patients $(96.5 \%)$ in the current study were maried and hence can be stratfied at low risk level but the patients who did not get married/ at right age are likely to become victim of breast cancer.

Income: Devlopment of breast cancer has been linked with income and education level of women in literature because it determins life style behavior and area of living. We have grouped our patients in to low, medium and high income class basing on different kind of employment/ work/ job done. About (17.5\%) current patients belonged to low education and low income level, which is near to californian patients where $9 \%$ of the patients were marked as "lower middle class" and hence were placed in low risk strata (Wrensch et al., 2003). Moreover, $71 \%$ of the existing patients were grouped in to medium income class compared to $55 \%$ of californian patients who were placed in same income class (Wrensch et al., 2003) whereas $11.5 \%$ patients belonged to the upper class and had high risk of breast cancer development.

\section{Place of residence}

Residential area has been cited as a risk factor in the development of breast cancer. It is observed that women living in urban areas are more vulnerable to this disease as compared to the women living in rural areas. Our results also support this notion since $60 \%$ of the patients belonged to urban areas and rest of the patients were living in rural places. Vorobiof (2001) has reported that place of living influences patients' decision to get early treatment or to evade the suggested medical remedies for cancer because people from rural places still incline to apply native ways of treatment.

\section{Parity}

Our data indicates that majority of the pateints $(80 \%)$ had one, two or few children which enlist them into a low risk category. These results are in agreement with the notion that multi-parity decreases endogenous oestrogen levels and hence ceases the level of risk (Fregene and Newman, 2005) while nulli-parity augment the level of prolactin which amplify the risk for breast cancer (Travis and Key, 2003). Risk for breast cancer diminishes for females who underwent delivery process for three or more times (Wrensch et al., 2003), while having few children increases risk (Sprague et al., 2008).

Breast feeding: Breast feeding shields females against breast cancer through numerous ways causing hormonal changes in the body and breast, late ovulation and excretion of carcinogenic agents (Travis and Key, 2003; Wrensch et al., 2003; Lord et al., 2008). Hormonal changes caused by breast feeding are linked with delayed reappearance of a new mother's menstrual periods and hence diminishes the likelyhood of exposure to hormones like oestrogen and risk for breast cancer (Clemons and Goss, 2001).

It is reported that breast feeding for approximately 16 months diminishes the risk of breast cancer in woman
(Fregene and Newman, 2005). Our data reflected that majority of the patients $(86.5 \%)$ in the current study had breastfed for 12 months or more and hence correspond to low risk level. Only $2 \%$ of the interviewed patients did not breastfeed their children.

Ethical Considerations: All ethical issues including plagiarism, Informed Consent, misconduct, data fabrication and/or falsification, double publication and/ or submission, redundancy, etc. have been completely observed by the author.

\section{Acknowledgements}

We thank the administration of INMOL, hospital Lahore, and director Division of Science and Technology, University of education, Lahore for extending cooperation to perform this study. The authors declare that there is no conflict of interests.

\section{References}

Abhidha M, Pamela AJ, Abhishek S, et al (2015). Passive Smoking and Breast Cancer - a Suspicious Link, Asian Pac J Cancer Prev, 16, 5715-9.

Abbasis S, Azimi C, Othman F, et al (2009). Risk factors for breast cancer in Iranian women, a case-control study. Int $J$ Cancer Res, 46, 132-38.

Anyanwu SN (2000). Breast cancer in Eastern Nigeria, a ten years review. West African J Med, 19, 120-5.

Berstad P, Coates RJ, Bernstein L, et al (2010). A case-control study of body mass index and breast cancer risk in white and African-American women. Cancer Epidemiol Biomarkers Prev, 19, 1532-44.

Bhikoo R, Srinivasa S, Yu TC, et al (2011). Systematic review of breast cancer biology in developing countries (part 2), Asian subcontinent and South East Asia. Cancers, 3, 2382-401.

Bhurgri Y, Bhurgri A, Hassan SH et al (2000). Cancer incidence in Karachi, Pakistan, first results from Karachi Cancer Registry. Int J Cancer, 85, 325-9.

Bhurgri Y (2004). Karachi cancer registry data--implications for the national cancer control program of pakistan. Asian Pac $J$ Cancer Prev, 5, 77-82.

Chen JG, Zhu J, Parkin D et al (2006). Trends in the incidence of cancer in Qidong, China. Int J Cancer, 119, 1447-54.

Chen Y, Thompson W, Semenciw R et al (1999). Epidemiology of contralateral breast cancer. Cancer Epidemiol Biomarkers Prev, 8, 855-61.

Clemons M, Goss P (2001). Estrogen and the risk of breast cancer. $N$ Engl J Med, 344, 276-85.

Ferlay J, Shin HR, Bray F, et al (2010). Estimates of worldwide burden of cancer in 2008, GLOBOCAN 2008. Int J Cancer, 127, 2893-917

Fregene A, Newman LA (2005). Breast cancer in sub-saharan africa, how does it relate to breast cancer in AfricanAmerican women? Cancer, 103, 1540-50.

Ferlay J, Shin HR, Bray F, et al (2010). GLOBOCAN 2008 v1.2, cancer incidence and mortality worldwide, IARC Cancer Base No. 10 [Internet]. Lyon, France, International Agency for Research on Cancer.

Kumar S, Imam AM, Manzoor NF et al (2009). Knowledge, attitude and preventive practices for breast cancer among health care professionals at Aga Khan Hospital, Karachi. $J$ Pak Med Association, 59, 474-78

Karapanou O, Papadimitriou A (2010). Determinants of menarche. Rep biol endoc, 8, 115-22. 
Lee BL, Liedke PE, Barrios CH, et al (2012). Breast cancer in Brazil, present status and future goals. Lancet Oncol, 13, 95-102.

Liede A, Malik IA, Aziz Z et al (2002). Contribution of BRCA1 and BRCA2 mutations to breast and ovarian cancer in Pakistan. Am J Hum Genet, 71, 595-606.

Lord SJ, Bernstein L, Johnson KA et al (2008). Breast cancer and hormone receptor status in older women by parity, age of first birth, and breastfeeding, A case-control study. Cancer Epidemiol Biomarkers Prev, 17, 1723-30.

Lundy J (1994). Prophylactic mastectomy, An assessment of the key factors involved in decision- making, In Breast cancer, Controversies in management. Ed by Wise L. and Johnson H. Armonk, New York, Futura Publishing Company. Inc, 271.

Mousavi SM, Mohaghegghi MA, Mousavi-Jerrahi A, et al (2006). Burden of breast cancer in Iran, a study of the Tehran population based cancer registry. Asian Pac J Cancer Prev, 16, 5049-52.

Norman P, Brain K (2005). An application of an extended health belief model to the prediction of breast self-examination among women with a family history of breast cancer. $\mathrm{Br} J$ Health Psychol, 10, 1-16.

Ogundiran TO, Huo D, Adenipekun A et al (2010). Case-control study of body size and breast cancer in Nigerian women. Am J Epidemiol, 172, 682-90

Parkin DM, Whelan S, Ferlay J et al (2005). Cancer incidence in five Continents.Vol.VIII IARC Scientific Publications No. 155. 150 Cours Albert Thomas, 69372 Lyon CEDEX 08, France.

Parkin DM, Bray F, Ferlay J et al (2005).Global cancer statistics, 2002. CA Cancer J Clin, 55, 74-108.

Porter PL (2009). Global trends in breast cancer incidence and mortality. Salud Publica Mex, 51, 141-6.

Puri S, Mangat C, Bhatia V et al (2009). Awareness of risk factors and aspects of breast cancer among North Indian women. Internet J Health, 8, 1-8.

Rambau PF, Chalya PL, Manyama MM et al (2011). Pathological features of breast cancer seen in North-western Tanzania, a nine years retrospective study. BMC Res Notes, 4, 214-19.

Sariego J (2010). Breast cancer in the young patient. Am Surg, 76, $1397-400$.

Sasco AJ. (2001). Epidemiology of breast cancer, an environmental disease? APMIS, 109, 321-32.

Sasieni PD, Shelton J, Ormiston-Smith N et al (2011). What is the lifetime risk of developing cancer? The effect of adjusting for multiple primaries. Br J Cancer, 105, 460-5.

Saweer AAL, Yacoub F, Mohammed N et al (2003). The prevalence of risk factors among women diagnosed with breast cancer. Bahrain Medical Bulletin, 25, 1-6.

Sprague BL, Trentham-Dietz A, Egan KM, et al (2008). Proportion of invasive breast cancer attributable to risk factors modifiable after menopause. Am J Epidemiol, 168, 404-11.

Travis RC, Key TJ (2003). Oestrogen exposure and breast cancer risk. Breast Cancer Res, 5, 239-47.

Vorobiof DA, Sitas F, Vorobiof G (2001). Breast cancer incidence in South Africa. J Clin Oncol, 19, 25-127.

Wrensch M, Chew T, Farren G et al (2003). Risk factors for breast cancer in a population with high incidence rates. Breast Cancer Res, 5, 88-102

Byrd-Bredbenner C, Beshgetoor D, Moe G (2012). Wardlaw's Perspectives in nutrition. $9^{\text {th }}$ edition, Published by McGrawHill, USA.

Zegeye DT, Megabia WB, Mulu A (2009). Age at menarche and the menstrual pattern of secondary school adolescents in Northwest Ethiopia. BMC Womens Health, 9, 1-8. 